\title{
Incorporating ATP into biomimetic catalysts for realizing exceptional enzymatic performance over a broad temperature range
}

\author{
Youhui Lin ${ }^{1,2}$, Yanyan Huang ${ }^{1,2}$, Jinsong $\operatorname{Ren}^{1,2}$ and Xiaogang $\mathrm{Qu}^{1,2}$ \\ There has been great interest in the development of artificial inorganic nanomaterials that mimic natural peroxidases. \\ Unfortunately, these nanomaterials usually possess relatively low catalytic activity and are generally considered to work \\ effectively only within a narrow temperature range (for example, they are inactive at high temperatures). Intriguingly, adenosine \\ triphosphate (ATP) is not only the ubiquitous energy currency of life, it is also known to form charge transfer complexes with \\ aromatic molecules and to participate in free radical redox chemistry. Inspired by its unique properties, for the first time, we \\ reveal a novel catalytic role for ATP and show that ATP is an ideal boosting agent for markedly improving the catalytic activity \\ of a peroxidase mimic over a broad temperature range and, more significantly, making it possible to achieve exceptionally \\ efficient high-temperature catalytic reactions. These observations pave the way for identifying highly effective modulators that \\ promote the overall performance of artificial enzymes.
}

NPG Asia Materials (2014) 6, e114; doi:10.1038/am.2014.42; published online 18 July 2014

\section{INTRODUCTION}

Natural enzymes, which are biological molecules of immense catalytic force and high substrate specificity, have attracted considerable attention for use in pharmaceutical processes, agrochemical production, biosensing and food industry applications. ${ }^{1-3}$ However, their applications are largely limited by their intrinsic properties, such as the sensitivity of catalytic activity to environmental conditions and low operational stability (owing to denaturation and digestion), as well as by the high costs of preparation and purification. ${ }^{4,5}$ To circumvent the aforementioned limitations, tremendous efforts have been made to develop biomimetic catalysts. Among the countless examples of artificial enzymes, the emergence of and recent advances in nanotechnology and biology provide new opportunities for designing functional nanomaterials with enzyme-like characteristics. ${ }^{4,6-8}$ These materials might serve as novel and promising artificial enzymes with the advantages of facile preparation, tunability of catalytic activity and high stability under stringent conditions. Owing to the excellent catalytic properties of these nanomaterials, Scrimin and co-workers ${ }^{7}$ called them 'nanozymes' in analogy to the nomenclature of catalytically active polymers (synzymes). Magnetic nanoparticles, ${ }^{4,9} \mathrm{CeO}_{2},{ }^{6,10-12} \mathrm{~V}_{2} \mathrm{O}_{5},{ }^{13,14}$ gold nanoparticles, ${ }^{7,8,15-18}$ $\mathrm{PtPd}-\mathrm{Fe}_{3} \mathrm{O}_{4},{ }^{19,20}$ carbon nanotubes, ${ }^{21,22}$ graphene oxide and graphene nanocomposites ${ }^{23,24}$ and other types of nanoparticle have been found to exhibit unique enzyme-like catalytic activities and have already shown promising applications in medicine, biotechnology and environmental chemistry.

Since the initial discovery of magnetic nanoparticle-based catalytic capability, a significant amount of effort has been focused on imitating peroxidase activity with various nanomaterials. ${ }^{4,13,19-24}$ Nevertheless, the binding affinity and substrate specificity of most nanomaterial-based peroxidase mimics remain lower than those of natural enzymes (Supplementary Scheme S1). ${ }^{25}$ The reason for such differences is that in the active site of natural enzymes, a pocket is available for substrate recognition and catalysis. ${ }^{26}$ Therefore, to increase the binding affinity and specificity, the exterior surfaces of nanozymes may be engineered with functional groups that are similar to those that are exposed in natural enzymes. ${ }^{27}$ Moreover, the present peroxidase mimics often suffer from two additional shortcomings (Supplementary Scheme S1): first, they exhibit relatively low catalytic activity and, second, they can work effectively only within a narrow temperature range. ${ }^{4,23}$ These two issues can severely affect the catalytic performance of nanozymes and limit their effectiveness in practical systems, greatly restricting the further development and application of these novel biomimetic catalysts. Alongside the exponential rise in the number of catalytically active nanomaterials in the past few decades, the search for convenient ways to improve their catalytic efficiency has only begun to emerge. Very recently, we observed positive effects of ionic liquids on peroxidase mimic-mediated high-temperature

${ }^{1}$ State Key Laboratory of Rare Earth Resources Utilization and Laboratory of Chemical Biology, Changchun Institute of Applied Chemistry, Chinese Academy of Science, Changchun, Jilin, China and ${ }^{2}$ Graduate School of University of Chinese Academy of Sciences, Beijing, China

Correspondence: Dr Professor J Ren or Dr Professor X Qu, State Key Laboratory of Rare Earth Resources Utilization and Laboratory of Chemical Biology, Changchun Institute of Applied Chemistry, Chinese Academy of Science, Renim Street \#5625, Changchun, Jilin 130022, China.

E-mail: jren@ciac.ac.cn or xqu@ciac.ac.cn

Received 2 December 2013; revised 18 March 2014; accepted 1 May 2014 
catalytic reactions by enhancing the thermal stability of the product. ${ }^{28}$ Although promising, ionic liquids as modulators suffer from several drawbacks: (1) ionic liquids could partly or even completely inhibit the catalytic activity of nanozymes because of their viscosity and their impact on catalysts that limits the scope of their practical application; (2) even if the chemical access to ionic liquids were straightforward, they are not commercially available; (3) they are not readily adaptable to general artificial enzymes because of their high ionic strength. Thus, it remains a substantial challenge to find new, convenient and effective methods to promote peroxidase-like activity over a wide range of temperatures (Supplementary Scheme S1).

In this study, we present a new strategy for promoting peroxidaselike activity over a broad temperature range by utilizing adenosine triphosphate (ATP) as an effective modulator (Scheme 1). Here, $\mathrm{Au}-\mathrm{SiO}_{2}$ nanoparticles, which have been investigated as a peroxidase mimic, are used as a model system to provide the 'proof-of-principle' verification of the concept. They possess relatively low catalytic activity; additionally, despite superior thermal stability, their catalytic performance as a function of temperature (especially at high temperature) was far below expectations. Given the outstanding versatility of nanozyme-based applications, ${ }^{4,13,19-24}$ there is a great need to develop peroxidase mimics that are able to exhibit high catalytic activity over a broad temperature range, particularly at high temperatures. With this goal in mind, we sought effective promoters to enhance this enzyme-like activity. As is well known, ATP is a biological coenzyme that is present in all known forms of life and it plays a key role in metabolism. It can be considered a 'helper molecule' in biochemical transformations. ${ }^{29}$ Intriguingly, we find that not only can it be used as a coenzyme for biological enzymes, but it can also be used for nano-catalysis. With the aid of ATP, the catalytic efficiency of peroxidase mimics is greatly increased over a broad temperature range; more significantly, it becomes possible to achieve exceptionally efficient high-temperature catalytic reactions. Our new findings pave the way for identifying highly effective modulators that improve the overall performance of artificial enzymes and, more importantly, for realizing some catalytic reactions that could not be conducted in the past.

\section{EXPERIMENTAL PROCEDURES}

\section{Reagents and materials}

ATP and 3,3,5,5-tetramethylbenzidine (TMB) were obtained from BB (Markham, ON, Canada). Horseradish peroxidase was obtained from Shanghai Sangon Biological Engineering Technology \& Services (Shanghai, China). 3-Aminopropyltriethoxysilane (APTES), sodium borohydride $\left(\mathrm{NaBH}_{4}\right)$ and $\mathrm{N}$-cetyltrimethylammonium bromide (CTAB) were purchased from Alfa Aesar
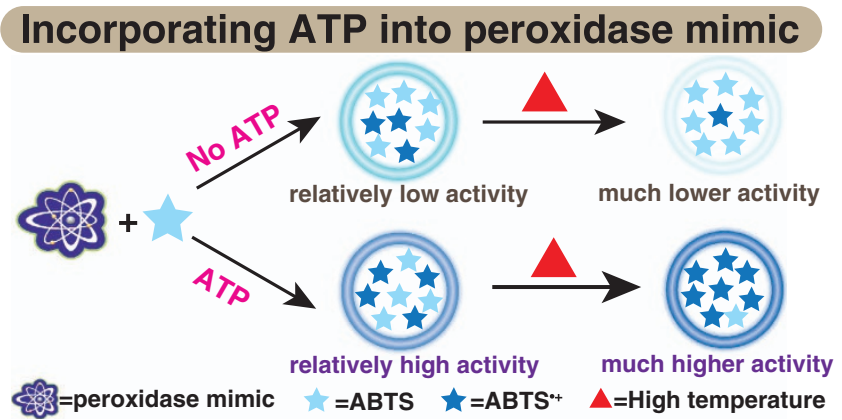

Scheme 1 Scheme showing promotion of catalytic activity within a fairly broad range of operating temperatures by incorporating adenosine triphosphate (ATP) with the peroxidase mimic.
(Tianjing, China). Adenosine $5^{\prime}$-[ $\gamma$-thio]triphosphate; $\geqslant 75 \%$ (ATP- $\gamma$-S), tetraethyl orthosilicate (TEOS), 2,2'-azino-bis(3-ethylbenzthiazoline-6-sulfonic acid (ABTS), sodium hydroxide and 1,3,5-trimethylbenzene were purchased from Sigma-Aldrich (St Louis, MO, USA). Malachite green hydrochloride was obtained from Aladdin (Shanghai, China). Hydrogen tetrachloroaurate (III) $\left(\mathrm{HAuCl}_{4} \cdot 3 \mathrm{H}_{2} \mathrm{O}\right)$ was purchased from Sinopharm Chemical Reagent Co. (Shanghai, China). $\mathrm{H}_{2} \mathrm{O}_{2}$ and ammonium molybdate were obtained from Beijing Chemicals (Beijing, China). All other reagents were of analytical reagent grade and were used as received. Ultrapure water (18.2 M $\Omega$; Millipore Co., Bedford, MA, USA) was used throughout the experiment.

\section{Measurements and characterization}

Transmission electron microscopy was performed using an FEI TECNAI G2 20 high-resolution transmission electron microscope operating at $200 \mathrm{kV}$. The UV-vis absorption spectra were obtained using a JASCO V-550 UV/Visible spectrophotometer (JASCO International Co., LTD, Tokyo, Japan). Electron paramagnetic resonance (EPR) spectra were recorded on a JES-FA 200 EPR spectrometer (JEOL, Tokyo, Japan). The instrument parameters were as follows: scanning frequency, $9.45 \mathrm{GHz}$; scanning power, $0.998 \mathrm{~mW}$; scanning temperature, $25^{\circ} \mathrm{C}$

\section{Preparation of $\mathrm{Au}-\mathrm{SiO}_{2}$ nanoparticles}

Mesoporous silica nanoparticles were prepared using a base-catalyzed sol-gel procedure. ${ }^{30}$ In order to avoid causing pore blockage after the encapsulation of functional nanoparticles, 1,3,5-trimethylbenzene was first used to swell the mesopores according to a slightly modified protocol described in the literature. ${ }^{31}$ Briefly, the as-synthesized silica nanoparticles $(0.50 \mathrm{~g})$ were dispersed in ethanol $(15 \mathrm{ml})$ by sonication for $30 \mathrm{~min}$, followed by the addition of $30 \mathrm{ml}$ of a 1:1 mixture $(\mathrm{v} / \mathrm{v})$ of deionized water and 1,3,5trimethylbenzene. The mixture was placed in an autoclave and held at $140{ }^{\circ} \mathrm{C}$ for $24 \mathrm{~h}$ without stirring. The resulting white powder was washed with ethanol and deionized water. To remove the CTAB, the white powder was refluxed for $16 \mathrm{~h}$ in a solution of $1.0 \mathrm{ml} \mathrm{HCl} \mathrm{(37 \% )} \mathrm{and} 50 \mathrm{ml}$ ethanol, followed by extensive washing with deionized water and methanol and drying under vacuum. As a result of this procedure, the mesopores were expanded to $\sim 3.1$ times their original size (expanding from a mean pore size of $2.8 \mathrm{~nm}$ to a mean pore size of $8.7 \mathrm{~nm}$ ), according to nitrogen sorption measurements (data not shown). Next, amine modification of the silica surface was performed by suspending the expanded silica nanoparticles $(200 \mathrm{mg})$ in a solution of APTES $(1 \mathrm{mmol})$ in dry toluene $(20 \mathrm{ml})$ and heating them under reflux for $24 \mathrm{~h} .^{32}$ The resulting nanoparticles were then collected by vacuum filtration, washed thoroughly with toluene and dried under vacuum.

Finally, $\mathrm{Au}-\mathrm{SiO}_{2}$ nanoparticles were synthesized by our recently developed method. ${ }^{33}$ Briefly, the above-mentioned nanoparticles $(100 \mathrm{mg})$ were dispersed in $10 \mathrm{ml}$ of distilled water by sonication for $10 \mathrm{~min}(100 \mathrm{mg})$, followed by the addition of the $\mathrm{HAuCl}_{4}$ solution $(1 \mathrm{ml} ; 20 \mathrm{~mm})$. After $10 \mathrm{~min}$ of sonication, freshly prepared $\mathrm{NaBH}_{4}(5 \mathrm{ml} ; 0.1 \mathrm{M})$ was added into the aqueous solution with vigorous stirring. After mixing, the resulting suspension was stirred for another $1 \mathrm{~h}$

\section{Peroxidase-like activity}

Enzymatic assays of the Au- $\mathrm{SiO}_{2}$ nanocatalyst were performed according to published procedures. ${ }^{5}$ Kinetic measurements were carried out in time course mode by monitoring the absorbance change at $417 \mathrm{~nm}$ for ABTS (or $652 \mathrm{~nm}$ for TMB) on a JASCO V-550 UV/Vis spectrophotometer. Experiments were carried out using $250 \mu \mathrm{g} \mathrm{ml}^{-1} \mathrm{Au}-\mathrm{SiO}_{2}$ catalyst in a reaction volume of $400 \mu \mathrm{l}$ $25 \mathrm{~mm}$ phosphate buffer ( $\mathrm{pH} 4.0$ ) with $1 \mathrm{~mm}$ ABTS (or TMB), $50 \mathrm{~mm} \mathrm{H}_{2} \mathrm{O}_{2}$ and $2.5 \mathrm{~mm}$ ATP unless otherwise stated.

\section{ATP hydrolysis and free inorganic phosphate determination} ATP $(2.5 \mathrm{~mm})$ was incubated in distilled water at different temperatures for $30 \mathrm{~min}$. The ATP solution at $4{ }^{\circ} \mathrm{C}$ was used as a blank. Then, the amount of inorganic phosphate released into solution was measured using a malachite green-ammonium molybdate assay, ${ }^{34}$ and the sample's absorbance was read at $620 \mathrm{~nm}$. Briefly, solutions of malachite green $(0.081 \% \mathrm{w} / \mathrm{v})$, polyvinyl alcohol $(2.3 \% \mathrm{w} / \mathrm{v})$ and ammonium heptamolybdate tetrahydrate $(5.7 \% \mathrm{w} / \mathrm{v}$ in $6 \mathrm{M}$ 
$\mathrm{HCl}$ ) were mixed with water in a ratio of 2:1:1:2. The obtained reagent solution $(320 \mu \mathrm{l})$ was dropped into the above-mentioned ATP solution $(100 \mu \mathrm{l})$. Next, $40 \mu \mathrm{l}$ of $34 \%$ sodium citrate was added to halt the hydrolysis of ATP. After mixing, the reaction was allowed to proceed for $15 \mathrm{~min}$ before performing spectroscopic measurements. In addition, a standard curve for phosphate was generated using $\mathrm{NaH}_{2} \mathrm{PO}_{4}$, and the amount of inorganic phosphate generated by ATP hydrolysis was calculated using the standard curve.

\section{RESULTS}

Catalysis with Au catalysts is a topic of great current interest. Because the catalytic properties of gold nanomaterials strongly depend on the particle size and stability, the use of $\mathrm{Au}$ as a catalyst requires careful preparation of the Au metal with a focus on achieving very small and stable Au particles. In our experiments, a high density of very small and stable AuNPs encapsulated in an expanded mesoporous silica support ( $\mathrm{Au}-\mathrm{SiO}_{2}$ nanoparticles) was synthesized using our recently developed method (see details in the Experimental procedures and Supplementary Figures S1 and S2). ${ }^{33}$ Next, the peroxidase-mimicking activity of the $\mathrm{Au}-\mathrm{SiO}_{2}$ nanocatalyst in solution was evaluated. Similar to the enzyme horseradish peroxidase (HRP), the $\mathrm{Au}-\mathrm{SiO}_{2}$ nanocatalyst was able to catalyze the reaction of the peroxidase substrate ABTS in the presence of $\mathrm{H}_{2} \mathrm{O}_{2}$ to produce a colored product, $\mathrm{ABTS}^{+}$, that was accompanied by an increase in the absorption peak at $417 \mathrm{~nm}$ (Figures 1a and b). Control experiments indicated that neither $\mathrm{H}_{2} \mathrm{O}_{2}$ nor catalyst alone could efficiently oxidize ABTS, as indicated by the absence of color development and absorbance increase. These results demonstrated that $\mathrm{Au}-\mathrm{SiO}_{2}$ nanoparticles could serve as an effective peroxidase mimic. Furthermore, $\mathrm{pH}$-dependent studies of the catalyzed oxidation of ABTS showed that as the $\mathrm{pH}$ value of the buffered solution increased from 4.0 to 7.0, the ability of the $\mathrm{Au}-\mathrm{SiO}_{2}$ catalyst to oxidize the substrate decreased (Supplementary Figure S3). Although the $\mathrm{Au}-\mathrm{SiO}_{2}$ catalyst has relatively low catalytic activity and binding affinity (Supplementary
Table S1), our artificial enzyme, as an inorganic nanomaterial, exhibits high $\mathrm{pH}$ and thermal stability compared with the natural HRP enzyme. As shown in Figure 1c, the activity of HRP was completely inhibited under heating $\left(85^{\circ} \mathrm{C}, 5 \mathrm{~min}\right)$ mainly because its own structure was destroyed, whereas the activity of the $\mathrm{Au}-\mathrm{SiO}_{2}$ catalyst remained almost the same as before heating. In addition, the pH stability of $\mathrm{Au}-\mathrm{SiO}_{2}$ and $\mathrm{HRP}$ was investigated. To test this, we first incubated both $\mathrm{HRP}$ and the $\mathrm{Au}-\mathrm{SiO}_{2}$ catalyst at a range of $\mathrm{pH}$ values (1-9) for $2 \mathrm{~h}$ and then measured their activities under standard conditions ( $\mathrm{pH} 4.0$ and $37^{\circ} \mathrm{C}$ ). The $\mathrm{Au}-\mathrm{SiO}_{2}$ catalyst was found to remain stable over a wide $\mathrm{pH}$ range, from 1 to 9 (Supplementary Figure S4). In contrast, HRP showed no activity after treatment at $\mathrm{pH}$ values lower than 2.5. These results further confirmed that natural enzymes can easily lose catalytic activity when the environment changes, whereas artificial enzymes have excellent stability under stringent conditions.

Because the $\mathrm{Au}-\mathrm{SiO}_{2}$ nanocatalyst exhibited superior thermal stability to the natural enzyme, we then expected that it would possess efficient catalytic activity over a fairly broad temperature range. Unfortunately, similar to HRP (Supplementary Figure S5) and other peroxidase mimics (for example, $\mathrm{Fe}_{3} \mathrm{O}_{4}$, graphene oxide), ${ }^{4,23}$ the $\mathrm{Au}-\mathrm{SiO}_{2}$ nanocatalyst only worked effectively within a narrow temperature range (that is, it was inactive at high temperature). As shown in Figure 2a, the reaction rate increases with temperature to a maximum level, then abruptly declines with further increases in temperature. Thus, the use of enzyme mimics has been limited by their low catalytic activity at high temperatures. However, it is well known that ATP is not only considered to be the ubiquitous energy currency of life, ${ }^{35}$ but is also well known to form charge transfer complexes with aromatic molecules and to participate in free radical redox chemistry. ${ }^{36-39}$ For instance, the positive effects of ATP on ribonucleotide reductase-mediated catalytic reactions have been reported. ${ }^{37}$ In addition, ATP could bind to an in vitro-selected

a
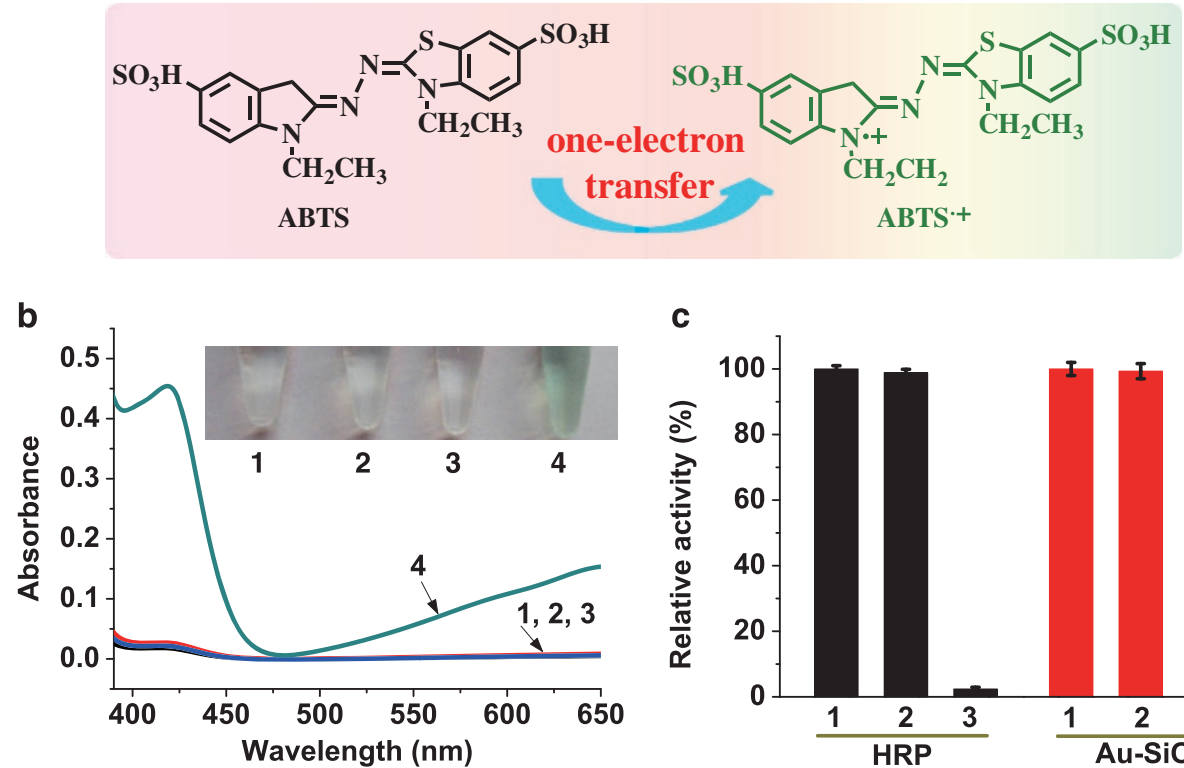

C

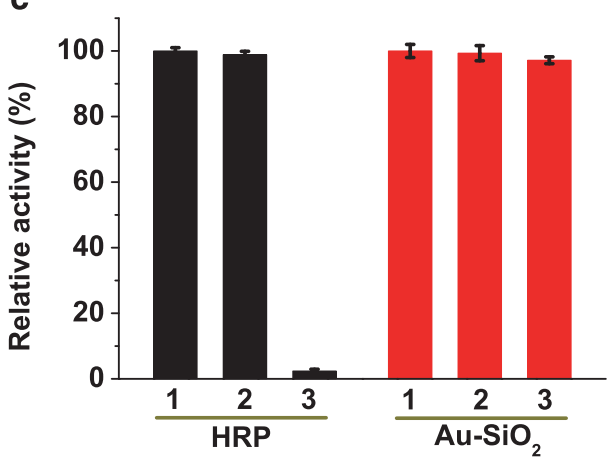

Figure 1 (a) The molecular structures of 2,2'-azino-bis(3-ethylbenzthiazoline-6-sulfonic acid (ABTS) and its one-electron oxidation product, ABTS + ${ }^{+}$, are shown. (b) Ultraviolet-visible (UV-vis) spectra and the corresponding visual color changes as a result of the catalyzed oxidation of ABTS in phosphate buffer $\left(20 \mathrm{~mm}, \mathrm{pH}\right.$ 4.0): 1 , blank control; 2, $\mathrm{H}_{2} \mathrm{O}_{2}$ alone; 3, Au-SiO ${ }_{2}$ catalyst alone; 4, $\mathrm{H}_{2} \mathrm{O}_{2}$ and Au-SiO $\mathrm{S}_{2}$ catalyst $\left(\mathrm{ABTS}=1 \mathrm{~mm}, \mathrm{H}_{2} \mathrm{O}_{2}=50 \mathrm{~mm}\right.$, $\mathrm{Au}-\mathrm{SiO}_{2}=250 \mu \mathrm{g} \mathrm{ml}{ }^{-1}$ ). (c) Effects of temperature on the thermal stability of horseradish peroxidase (HRP) and Au- $\mathrm{SiO}_{2}$ catalyst. $1, \mathrm{Blank}$ control; $2,4{ }^{\circ} \mathrm{C}$, $24 \mathrm{~h}$ storage; $3,85^{\circ} \mathrm{C}, 5 \mathrm{~min}$ heating. 

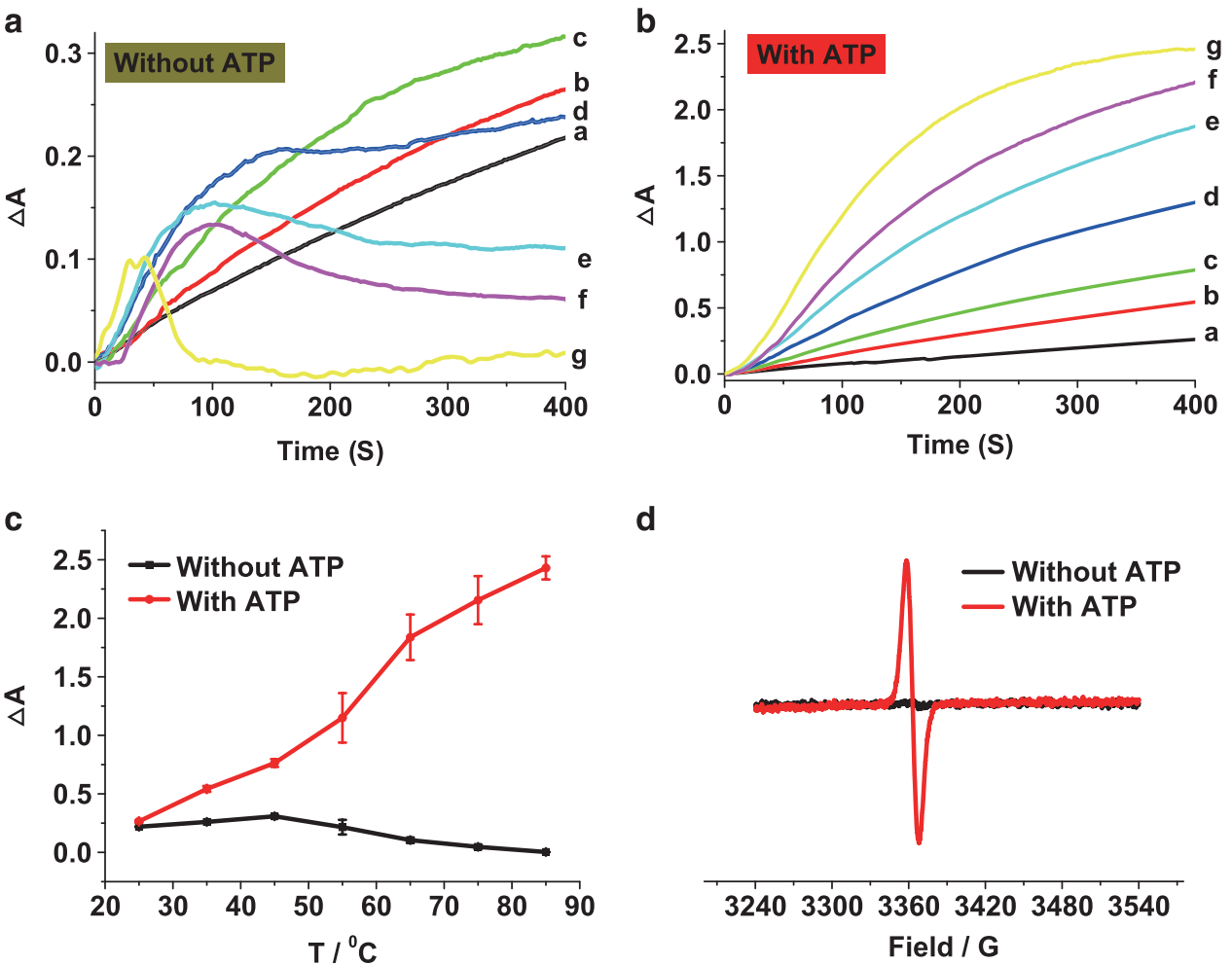

Figure 2 (a, b) Time-dependent absorbance changes at $417 \mathrm{~nm}$ as a function of operating temperature in the absence or presence of $2.5 \mathrm{~mm}$ ATP (a-g: 25 , $35,45,55,65,75$ and $85^{\circ} \mathrm{C}$ ). (c) The corresponding absorption values at $417 \mathrm{~nm}$ after $400 \mathrm{~s}$. (d) Electron paramagnetic resonance (EPR) spectra obtained after the catalyzed oxidation of 2,2'-azino-bis(3-ethylbenzthiazoline-6-sulfonic acid (ABTS) under different conditions: (1) Au-SiO ${ }_{2}$ and $\mathrm{H}_{2} \mathrm{O}_{2}$, $85^{\circ} \mathrm{C}$; (2) Au- $\mathrm{SiO}_{2}, \mathrm{H}_{2} \mathrm{O}_{2}$ and adenosine triphosphate (ATP), $85^{\circ} \mathrm{C}$. (ABTS $=1 \mathrm{~mm}, \mathrm{ATP}=2.5 \mathrm{~mm}, \mathrm{H}_{2} \mathrm{O}_{2}=50 \mathrm{~mm}, \mathrm{Au}-\mathrm{SiO}_{2}=250 \mu \mathrm{gml}-1$ ).

27-base anti-ATP aptamer with high affinity and could induce the aptamer to undergo a conformational change from random coil to tertiary structure. ${ }^{40}$ Inspired by the unique properties of ATP, we found that ATP could promote the catalytic activity of the $\mathrm{Au}-\mathrm{SiO}_{2}$ catalyst within a fairly broad range of operating temperatures (Figures $2 \mathrm{~b}$ and $\mathrm{c}$ ). As shown in Figure $2 \mathrm{c}$, the activity of the nanocatalyst shows a linear increase along with the temperature elevation from 25 to $85^{\circ} \mathrm{C}$, and this is in sharp contrast to that obtained without ATP (Figures 2a and c) and to that of the HRP enzyme (Supplementary Figure S5). More importantly, at high temperature, the activity of the nanocatalyst combined with ATP was much greater than that in the absence of ATP and that of HRP. For instance, an $>200$-fold increase of the peroxidase-like activity occurred in the presence of $2.5 \mathrm{~mm}$ ATP compared with that without ATP at $85^{\circ} \mathrm{C}$. Furthermore, the reaction rate at high temperature increased with increasing ATP concentration (Supplementary Figure S6), indicating that the enhanced activity was strongly dependent on ATP concentration. In addition, because $\mathrm{ABTS}^{-+}$, a semi-stable radical, can be directly identified from the magnetic parameters of the EPR spectrum, we used the EPR technique to study the peroxidase-like activity at high temperature. ${ }^{41}$ In samples without ATP, the characteristic EPR spectra of the reaction solution were weak (Figure 2d). In contrast, strong EPR spectra for ABTS $+{ }^{+}$appeared upon the addition of ATP. These EPR results are fully consistent with the absorbance assay (Figure 2c) and strongly support our data.

To further demonstrate the ability of ATP to enhance catalytic efficiency at high temperature, another experiment was performed (Supplementary Figure S7) in which ATP was added to an $\mathrm{Au}-\mathrm{SiO}_{2}-$ catalyzed $\mathrm{ABTS} / \mathrm{H}_{2} \mathrm{O}_{2}$ system after the reaction proceeded without ATP for $400 \mathrm{~s}$. Without ATP, the catalytic activity of the nanocatalyst at high temperature was very low, whereas when ATP was added, a remarkable increase of catalytic activity was observed. Similarly, the positive effects of ATP were also suitable for another peroxidase substrate, TMB; that is, much higher activity was observed for TMB in the presence of ATP than that in the absence of ATP (Supplementary Figure S8). Taken together, the catalytic reactions could be improved over a broad range of operating temperatures by combining ATP with the thermally stable $\mathrm{Au}-\mathrm{SiO}_{2}$ catalyst.

Next, the effects of other nucleotides, ATP analogs and radical scavengers on the catalytic system were investigated. Nucleotides were divided into two groups. One group was ATP, ADP and AMP that differ in the number of phosphate groups. In this group, only ATP showed positive effects on the catalytic reaction at high temperature that indicated the importance of the phosphate groups (Supplementary Figure S9). The other group included ATP, CTP, GTP and UTP that differ in the nucleotide base. ATP, GTP and CTP showed positive effects on the catalytic reaction, and UTP had no effect on the catalytic reaction. These results imply that the nucleobase also plays an important role in the reaction-promoting ability. In addition, radical scavengers (for example, glutathione and ascorbic acid) and the non-hydrolyzable ATP analog ATP- $\gamma-S$ could not function as effective additives because they could cause reduction of $\mathrm{ABTS}^{+}+$or oxTMB (Supplementary Figure S10).

\section{DISCUSSION}

Although the presence of ATP greatly enhanced the catalytic performance of the $\mathrm{Au}-\mathrm{SiO}_{2}$ catalyst, an explanation was needed concerning the source of the enhanced catalysis in this system. First of all, it was very important to rule out the possibility that the ATP itself promoted the activity. To test this, we incubated ATP in the reaction 
a

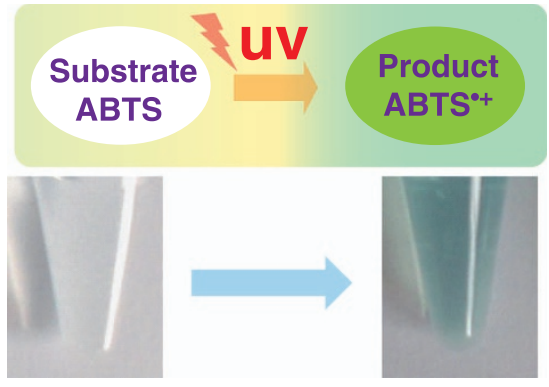

C

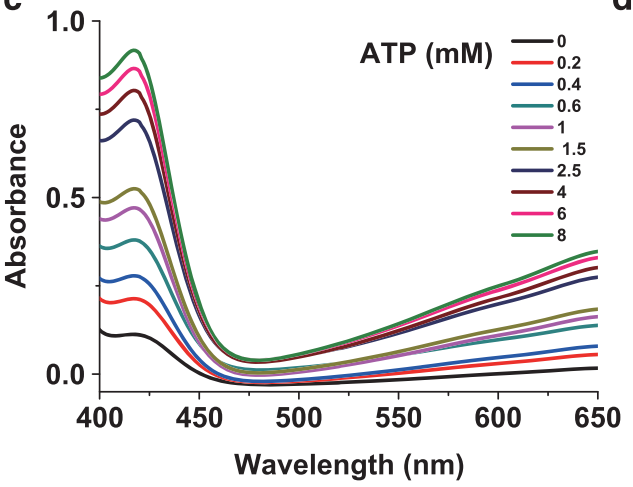

b

d
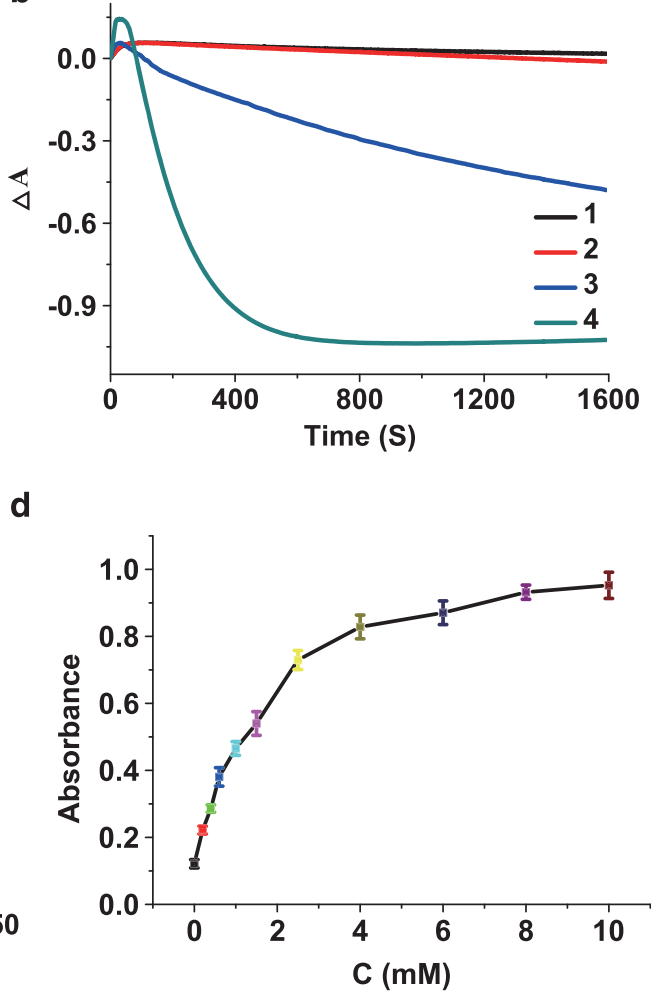

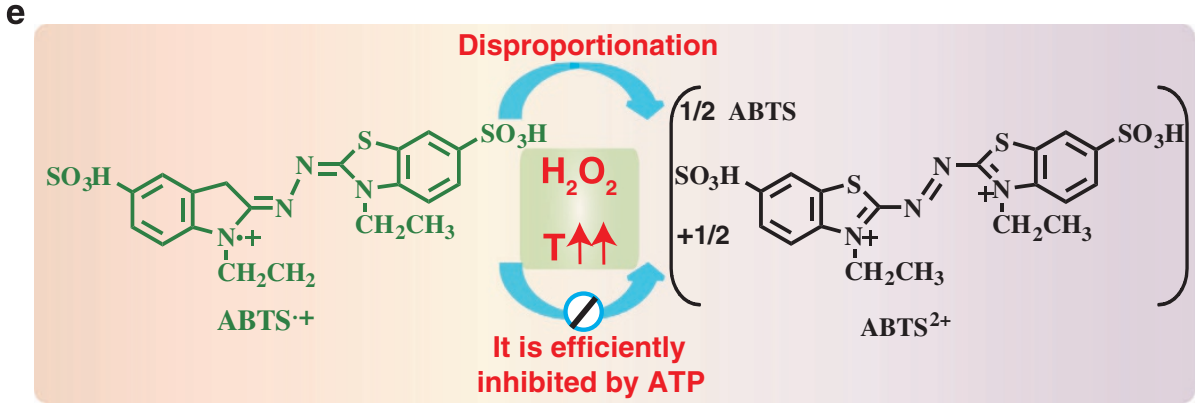

Figure 3 (a) Production of ABTS + by direct photolysis of 2,2'-azino-bis(3-ethylbenzthiazoline-6-sulfonic acid (ABTS) under ultraviolet (UV) irradiation. (b) The stability of ABTS ${ }^{+}$under different conditions: $1,37^{\circ} \mathrm{C} ; 2,50 \mathrm{~mm} \mathrm{H}_{2} \mathrm{O}_{2}, 37^{\circ} \mathrm{C} ; 3,85^{\circ} \mathrm{C}$; and $4,50 \mathrm{~mm} \mathrm{H}_{2} \mathrm{O}_{2}, 85^{\circ} \mathrm{C}$. (c) $\mathrm{Ultraviolet}^{-v i s i b l e}$ (UV-vis) spectra of ABTS + after heating to $85^{\circ} \mathrm{C}$ in the presence of $60 \mathrm{~mm} \mathrm{H}_{2} \mathrm{O}_{2}$ and increasing adenosine triphosphate (ATP) concentration (0-8 mM). (d) Plots of the absorption values at $417 \mathrm{~nm}$ as a function of the ATP concentration. (e) Disproportionation of the product, ABTS ${ }^{+}$, can be efficiently inhibited by ATP.

Table 1 Comparison of adenosine triphosphate (ATP) and ionic liquid as modulators in an artificial enzyme system

\begin{tabular}{ll}
\hline Modulator & Property \\
\hline ATP & 1. Promotes activity over a broad temperature range \\
& 2. Easily adaptable to other artificial enzymes \\
& 3. Cheap and commercially available \\
Ionic liquid ${ }^{28}$ & 1. Inhibits activity at a relatively low temperature \\
& 2. Not adaptable to many other catalysts \\
& 3. Relatively expensive
\end{tabular}

Here, we reveal a novel catalytic role of ATP and show that ATP is an ideal boosting agent to markedly increase the activity of a peroxidase mimic over a broad temperature range and, in particular, to promote efficient high-temperature catalytic reactions. These observations pave the way for the development of modulators for promoting the overall performance of artificial enzymes. solution (including ABTS and $\mathrm{H}_{2} \mathrm{O}_{2}$ ) for $400 \mathrm{~s}$ at 37 or $85^{\circ} \mathrm{C}$. As shown in Supplementary Figure S11, the absorption signal was very weak, indicating that ATP alone had no peroxidase-like activity and that the increased activity was because of the boosting impact of ATP on the biomimetic catalyst. In addition, previous work revealed that the enzymatic product $\left(\mathrm{ABTS}^{+}{ }^{+}\right.$) is relatively unstable in aqueous solution and rapidly decays to a colorless product through disproportionation. ${ }^{42}$ This reaction is particularly accelerated when a high concentration of $\mathrm{H}_{2} \mathrm{O}_{2}$ is used. Based on these observations, it was essential to study the thermal stability of the reaction product with and without $\mathrm{H}_{2} \mathrm{O}_{2}$ during heating. Initially, ABTS was directly photolyzed by ultraviolet (UV) irradiation to yield the one-electron oxidized radical ABTS ${ }^{+}$(Figure $\left.3 \mathrm{a}\right) .{ }^{43}$ Then, to test the stability of the product, we performed an experimental study of the timedependent absorption change under different conditions. As shown 
in Figure 3b, at relatively low temperature, the absorption signal remained almost unchanged in the absence or presence of $\mathrm{H}_{2} \mathrm{O}_{2}$ (curves 1 and 2), indicating that $\mathrm{ABTS}^{+}+$was stable under these conditions. However, a decrease in the UV-vis signal was observed at relatively high temperature (curve 3 ) that was attributed to the disproportionation of ABTS ${ }^{+}$. Meanwhile, the disproportionation could be further accelerated by $\mathrm{H}_{2} \mathrm{O}_{2}$ (curve 4) that was demonstrated by the fast decrease of the absorption signal. Therefore, the thermal stability of the product plays a vital role in high-temperature catalysis.

Inspired by the recent reports from Monchaud and colleagues ${ }^{35}$ and Kong et al., ${ }^{39}$ we assumed that ATP could also be used as an effective modulator to inhibit disproportionation and stabilize cationic free radical ABTS ${ }^{+}$at high temperature. ${ }^{35,39}$ To evaluate the feasibility of our presumption, we explored the effects of ATP concentration on the thermal stability of the reaction product in the presence of $60 \mathrm{~mm} \mathrm{H}_{2} \mathrm{O}_{2}$. As shown in Figures $3 \mathrm{c}$ and $\mathrm{d}$, the disproportionation of ABTS ${ }^{+}$is gradually inhibited with increasing ATP concentration. We then used EPR to study the thermal stability of the enzymatic product. The EPR signal intensity decreased considerably in the absence of ATP (Supplementary Figure S12). In contrast, when ATP was added, the signal of ABTS ${ }^{+}$largely remained as a result of the ability of ATP to inhibit disproportionation of the product. Overall, these observations indicated that ATP could act as a stabilizing agent to improve thermal stability even in the presence of a high concentration of $\mathrm{H}_{2} \mathrm{O}_{2}$ (Figure 3e). Moreover, because the product is stable at relatively low temperatures (Figure 3b), the unexpected promotion of activity under these conditions indicates that other factors also contribute to the positive effects of ATP. Previous reports indicated that ATP may mimic the distal histidine residue of the peroxidase enzyme. ${ }^{35}$ In addition, our results demonstrated that the non-enzymatic hydrolysis of ATP could take place under our experimental conditions (Supplementary Figure S13). Altogether, the enhanced activity may be related to ATP as an $\mathrm{H}_{2} \mathrm{O}_{2}$ activator and electron transfer energizer during the catalytic process. ${ }^{35}$ Although the detailed mechanism for the boosting effect of ATP remains unclear, all of the results described above confirmed our hypothesis and indicated that ATP plays a very positive role in boosting catalytic processes over a wide range of temperatures.

We believe that the use of ATP as a modulator offers a facile but highly effective way to achieve exceptional peroxidase-like activity, and it shows three principal advantages over ionic liquids (Table 1). (1) The boosting effect of ATP is much more efficient than that of ionic liquids over a broad temperature range. In particular, ionic liquids have a negative effect on enzymatic activity at relatively low temperature. (2) ATP is easily adaptable to other artificial enzymes, whereas many nanomaterials become unstable when exposed to the high ionic strength of ionic liquids (Supplementary Figure S14). (3) Compared with ionic liquids, ATP is cheaper, more convenient and is commercially available. Therefore, our strategy greatly extends the range of applications for peroxidase mimics.

In summary, we have successfully used ATP as a boosting agent in an artificial enzyme system to achieve exceptional peroxidase-like activity over a broad temperature range. ATP is an ideal modulator not only because it is commercially available, cheap and convenient to use, but also because it has a high boosting ability and is easily adaptable to use with other nanozymes. Because ATP can stabilize the oxidation product and serve as an $\mathrm{H}_{2} \mathrm{O}_{2}$ activator and electron transfer energizer, the incorporation of ATP into peroxidase mimics offers a convenient way to circumvent the drawbacks of existing biomimetic catalysts (that is, low catalytic activity and a narrow range of operating temperatures). More importantly, with the aid of ATP, artificial enzymes can realize high-temperature catalytic reactions that could not be conducted previously. Overall, these findings form an important step toward developing highly effective modulators to improve the performance of existing biomimetic catalysts and, more significantly, to broaden the application of artificial nanomaterials.

\section{ACKNOWLEDGEMENTS}

We acknowledge financial support from the National Basic Research Program of China (Grants 2012CB720602 and 2011CB936004) and the National Natural Science Foundation of China (Grants 21210002 and 91213302).

1 Barber, J. Photosynthetic energy conversion: natural and artificial. Chem. Soc. Rev. $\mathbf{3 8}$, 185-196 (2009)

2 Breslow, R. Biomimetic chemistry and artificial enzymes: catalysis by design. Acc Chem. Res. 28, 146-153 (1995).

3 Hult, K. \& Berglund, P. Engineered enzymes for improved organic synthesis. Curr. Opin. Biotechnol. 14, 395-400 (2003).

4 Gao, L., Zhuang, J., Nie, L., Zhang, J., Zhang, Y., Gu, N., Wang, T., Feng, J., Yang, D., Perrett, S. \& Yan, X. Intrinsic peroxidase-like activity of ferromagnetic nanoparticles. Nat. Nanotechnol. 2, 577-583 (2007).

5 Xie, J., Zhang, X., Wang, H., Zheng, H. \& Huang, Y. Analytical and environmental applications of nanoparticles as enzyme mimetics. TrAC, Trends Anal. Chem. 39, 114-129 (2012)

6 Asati, A., Santra, S., Kaittanis, C., Nath, S. \& Perez, J. M. Oxidase-like activity of polymer-coated cerium oxide nanoparticles. Angew. Chem. Int. Ed. 8, 2308-2312 (2009).

7 Manea, F., Houillon, F. B., Pasquato, L. \& Scrimin, P. Nanozymes: gold-nanoparticlebased transphosphorylation catalysts. Angew. Chem. Int. Ed. 43, 6165-6169 (2004).

8 Pengo, P., Polizzi, S., Pasquato, L. \& Scrimin, P. Carboxylate-imidazole cooperativity in dipeptide-functionalized gold nanoparticles with esterase-like activity. J. Am. Chem. Soc. 127, 1616-1617 (2005).

9 Liu, S., Lu, F., Xing, R. \& Zhu, J.-J. Structural effects of Fe304 nanocrystals on peroxidase-like activity. Chem. Eur. J. 17, 620-625 (2011).

10 Asati, A., Kaittanis, C., Santra, S. \& Perez, J. M. pH-Tunable oxidase-like activity of cerium oxide nanoparticles achieving sensitive fluorigenic detection of cancer biomarkers at neutral pH. Anal. Chem. 83, 2547-2553 (2011).

11 Heckert, E. G., Karakoti, A. S., Seal, S. \& Self, W. T. The role of cerium redox state in the SOD mimetic activity of nanoceria. Biomaterials 29, 2705-2709 (2008).

12 Singh, S., Dosani, T., Karakoti, A. S., Kumar, A., Seal, S. \& Self, W. T. A phosphatedependent shift in redox state of cerium oxide nanoparticles and its effects on catalytic properties. Biomaterials 32, 6745-6753 (2011).

13 André, R., Natálio, F., Humanes, M., Leppin, J., Heinze, K., Wever, R., Schröder, H. C. Müller, W. E. G. \& Tremel, W. V2O5 Nanowires with an intrinsic peroxidase-like activity. Adv. Funct. Mater. 21, 501-509 (2011).

14 Natalio, F., André, R., Hartog, A. F., Stoll, B., Jochum, K. P., Wever, R. \& Tremel, W. Vanadium pentoxide nanoparticles mimic vanadium haloperoxidases and thwart biofilm formation. Nat. Nanotechnol. 7, 530-535 (2012).

15 Comotti, M., Della Pina, C., Matarrese, R. \& Rossi, M. The catalytic activity of "naked" gold particles. Angew. Chem. Int. Ed. 43, 5812-5815 (2004).

16 Comotti, M., Della Pina, C., Falletta, E. \& Rossi, M. Aerobic oxidation of glucose with gold catalyst: hydrogen peroxide as intermediate and reagent. Adv. Synth. Catal. 348, 313-316 (2006).

17 Luo, W., Zhu, C., Su, S., Li, D., He, Y., Huang, Q. \& Fan, C. Self-catalyzed, self-limiting growth of glucose oxidase-mimicking gold nanoparticles. ACS Nano 4, 7451-7458 (2010).

18 Zheng, X., Liu, Q., Jing, C., Li, Y., Li, D., Luo, W., Wen, Y., He, Y., Huang, Q., Long, Y. T. \& Fan, C. Catalytic gold nanoparticles for nanoplasmonic detection of DNA hybridization. Angew. Chem. Int. Ed. 50, 11994-11998 (2011).

19 Sun, X., Guo, S., Chung, C.-S., Zhu, W. \& Sun, S. A sensitive H2O2 assay based on dumbbell-like PtPd-Fe304 nanoparticles. Adv. Mater. 25, 132-136 (2013).

20 Sun, X., Guo, S., Liu, Y. \& Sun, S. Dumbbell-like PtPd-Fe304 nanoparticles for enhanced electrochemical detection of H2O2. Nano Lett. 12, 4859-4863 (2012).

21 Song, Y. J., Wang, X., Zhao, C., Qu, K., Ren, J. \& Qu, X. Label-free colorimetric detection of single nucleotide polymorphism by using single-walled carbon nanotube intrinsic peroxidase-like activity. Chem.-Eur. J 16, 3617-3621 (2010).

22 Cui, R., Han, Z. \& Zhu, J.-J. Helical carbon nanotubes: intrinsic peroxidase catalytic activity and its application for biocatalysis and biosensing. Chem. Eur. J. 17, 9377-9384 (2011)

23 Song, Y., Qu, K., Zhao, C., Ren, J. \& Qu, X. Graphene oxide: intrinsic peroxidase catalytic activity and its application to glucose detection. Adv. Mater. 22, 2206-2210 (2010).

24 Xue, T., Jiang, S., Qu, Y., Su, Q., Cheng, R., Dubin, S., Chiu, C. Y., Kaner, R., Huang, Y. \& Duan, X. Graphene-supported hemin as a highly active biomimetic oxidation catalyst. Angew. Chem. Int. Ed. 51, 3822-3825 (2012). 
25 Dong, Z., Luo, Q. \& Liu, J. Artificial enzymes based on supramolecular scaffolds. Chem. Soc. Rev. 41, 7890-7908 (2012).

26 Lin, Y., Ren, J. \& Qu, X. Catalytically active nanomaterials: a promising candidate for artificial enzymes. Acc. Chem. Res. 47, 1097-1105 (2014).

27 Kotov, N. A. Inorganic nanoparticles as protein mimics. Science 330, 188-189 (2010).

28 Lin, Y., Zhao, A., Tao, Y., Ren, J. \& Qu, X. Ionic liquid as an efficient modulator on artificial enzyme system: toward the realization of high-temperature catalytic reactions. J. Am. Chem. Soc. 135, 4207-4210 (2013).

29 Kalckar, H. M. The nature of energetic coupling in biological syntheses. Chem. Rev. 28, 71-178 (1941).

30 Huh, S., Wiench, J. W., Yoo, J. C., Pruski, M. \& Lin, V. S. Y. Organic functionalization and morphology control of mesoporous silicas via a co-condensation synthesis method. Chem. Mater. 15, 4247-4256 (2003).

31 Kim, M.-H., Na, H. K., Kim, Y. K., Ryoo, S. R., Cho, H. S., Lee, K. E., Jeon, H., Ryoo, R. \& Min, D. H. Facile synthesis of monodispersed mesoporous silica nanoparticles with ultralarge pores and their application in gene delivery. ACS Nano 5, 3568-3576 (2011).

32 Angelos, S., Khashab, N. M., Yang, Y. W., Trabolsi, A., Khatib, H. A., Stoddart, J. F. \& Zink, J. I. pH clock-operated mechanized nanoparticles. J. Am. Chem. Soc. 131, 12912-12914 (2009)

33 Lin, Y., Li, Z., Chen, Z., Ren, J. \& Qu, X. Mesoporous silica-encapsulated gold nanoparticles as artificial enzymes for self-activated cascade catalysis. Biomaterials 34, 2600-2610 (2013).

34 Motomizu, S., Wakimoto, T. \& Toei, K. Spectrophotometric determination of phosphate in river waters with molybdate and malachite green. Analyst 108, 361-367 (1983).

35 Stefan, L., Denat, F. \& Monchaud, D. Insights into how nucleotide supplements enhance the peroxidase-mimicking DNAzyme activity of the G-quadruplex/hemin system. Nucleic Acids Res. 40, 8759-8772 (2012)

36 Blocker, R. \& Roth, J. S. The reduction of uridine 5'-diphosphate and uridine 5'-triphosphate in some transplantable rat hepatomas. Cancer Res. 37, 1923-1928 (1977).
37 Jordan, A. \& Reichard, P. Ribonucleotide reductases. Annu. Rev. Biochem. 67, 71-98 (1998).

38 Jordan, A., Torrents, E., Sala, I., Hellman, U., Gibert, I. \& Reichard, P. Ribonucleotide reduction in Pseudomonas species: Simultaneous presence of active enzymes from different classes. J. Bacteriol. 181, 3974-3980 (1999).

39 Kong, D.-M., Xu, J. \& Shen, H.-X. Positive effects of ATP on G-Quadruplex-Hemin DNAzyme-mediated reactions. Anal. Chem. 82, 6148-6153 (2010).

40 Pei, H., Zuo, X., Pan, D., Shi, J., Huang, Q. \& Fan, C. Scaffolded biosensors with designed DNA nanostructures. NPG Asia Mat. 5, e51 (2013).

$41 \mathrm{He}$, W., Zhou, Y. T., Wamer, W. G., Hu, X., Wu, X., Zheng, Z., Boudreau, M. D. \& Yin, J. $J$ J. Intrinsic catalytic activity of Au nanoparticles with respect to hydrogen peroxide decomposition and superoxide scavenging. Biomaterials 34, 765-773 (2013).

$42 \mathrm{Li}, \quad$ T., Dong, S. \& Wang, E. G-Quadruplex aptamers with peroxidase-like DNAzyme functions: which is the best and how does it work? Chem. Asian J. 4, 918-922 (2009).

43 Lee, C. \& Yoon, J. UV direct photolysis of 2,2'-azino-bis(3-ethylbenzothiazoline-6sulfonate) (ABTS) in aqueous solution: Kinetics and mechanism. J. Photochem. Photobiol. 197, 232-238 (2008).

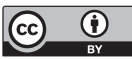

This work is licensed under a Creative Commons Attribution 3.0 Unported License. The images or other third party material in this article are included in the article's Creative Commons license, unless indicated otherwise in the credit line; if the material is not included under the Creative Commons license, users will need to obtain permission from the license holder to reproduce the material. To view a copy of this license, visit http:// creativecommons.org/licenses/by/3.0/

Supplementary Information accompanies the paper on the NPG Asia Materials website (http://www.nature.com/am) 